\title{
PARTITION IDENTITIES AND LABELS FOR SOME MODULAR CHARACTERS
}

\author{
G. E. ANDREWS, C. BESSENRODT, AND J. B. OLSSON
}

\begin{abstract}
In this paper we prove two conjectures on partitions with certain conditions. A motivation for this is given by a problem in the modular representation theory of the covering groups $\widehat{S}_{n}$ of the finite symmetric groups $S_{n}$ in characteristic 5 . One of the conjectures (Conjecture B below) has been open since 1974, when it was stated by the first author in his memoir [A3]. Recently the second and third author (jointly with A. O. Morris) arrived at essentially the same conjecture from a completely different direction. Their paper [BMO] was concerned with decomposition matrices of $\widehat{S}_{n}$ in characteristic 3 . A basic difficulty for obtaining similar results in characteristic 5 (or larger) was the lack of a class of partitions which would be "natural" character labels for the modular characters of these groups. In this connection two conjectures were stated (Conjectures $A$ and $B *$ below), whose solutions would be helpful in the characteristic 5 case. One of them, Conjecture $\mathrm{B}^{*}$, is equivalent to the old Conjecture B mentioned above. Conjecture $A$ is concerned with a possible inductive definition of the set of partitions which should serve as the required labels.
\end{abstract}

In $\S 1$ we give a brief description of the groups $\widehat{S}_{n}$ and their representations, leading up to Conjectures $\mathrm{A}$ and $\mathrm{B}^{*}$ as they were formulated in [BMO]. That section also presents the background for Conjecture $B$ as stated in [A3] and the equivalence of Conjectures $B$ and $B{ }^{*}$ is explained. Sections 2 and 3 are devoted to the proof of Conjecture B, and $\S 4$ to the proof of Conjecture $A$.

\section{THE CONJECTURES AND THEIR BACKGROUND}

For facts concerning the general representation theory of finite groups needed in the following, the reader is referred to [F, NT].

In 1911 Schur [S1] proved that the finite symmetric groups $S_{n}$ have covering groups $\widehat{S}_{n}$ of order $2\left|S_{n}\right|=2 \cdot n$ ! This means that there is an exact sequence

$$
1 \rightarrow\langle z\rangle \rightarrow \widehat{S}_{n} \rightarrow S_{n} \rightarrow 1
$$

where $\langle z\rangle$ is a central subgroup of order 2 in $\widehat{S}_{n}$. Then the irreducible representations of $\widehat{S}_{n}$ are divided in two categories:

Those representations which have $z$ in their kernel will be referred to as ordinary representations (in characteristic 0 ) and modular representations (in characteristic $p>0$ ).

Received by the editors August 25, 1992.

1991 Mathematics Subject Classification. Primary 05E10, 11 P83; Secondary 05A15. 
Those representations which do not have $z$ in their kernel will be referred to as spin representations (in characteristic 0 ) and modular spin representations (in characteristic $p>n$ ).

It is weil known that the ordinary irreducible representations of $\widehat{S}_{n}$ are labelled by the partitions of $n$. The modular irreducible representations of $\widehat{S}_{n}$ are labelled by the $p$-regular partitions of $n$, i.e. partitions where no part is repeated $p$ or more times. Both of these labellings are natural in several respects. One illustration of this is the following result of G. James (see [JK, 6.3.50, 6.3.60]). If an irreducible ordinary or modular representation is identified with its label, then the decomposition matrices have the form

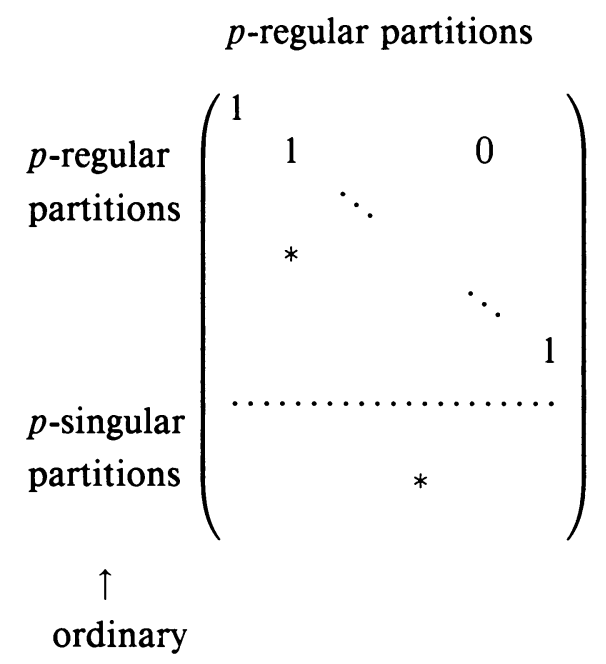

with the upper square matrix lower unitriangular.

Before proceeding to the spin case it should be mentioned that by a result of R. Brauer the number of modular irreducible representations of a finite group (over a splitting field) equals the number of conjugacy classes of elements in the group of order prime to $p$. Due to the well-known parametrization of the conjugacy classes of $S_{n}$ by partitions of $n$, the following result of Glaisher is used in the proof of (1):

The number of $p$-regular partitions of $n$ equals the number of partitions of $n$ into parts which are all prime to $p$.

Therefore Conjecture B* below may be seen as a "spin version" of Glaisher's result for $p=5$ !

In the spin case examples indicate that a result somewhat similar to (1) may hold apart from slight complications due to the appearance of associate characters (for $p>2$ ). Already Schur [S1] showed that the (associate classes of) spin representation of $\widehat{S}_{n}$ are labelled canonically by the partitions of $n$ into distinct parts. However it is still an open and apparently very difficult question to determine a class of partitions providing canonical labels for the (associate classes of) modular spin characters. For $p=3$ this question was solved in [BMO] and an analogue of (1) was proved.

James' proof of (1) features a systematic use of an $r$-inducing process, which makes it possible to build a class of partitions inductively as labels for the modular characters. This class of partitions is described in terms of a certain 
ladder condition and it is shown to be equal to the class of $p$-regular partitions. Conjecture A below may be seen as a "spin version" of this for $p=5$ and "ladders" will occur in its proof in $\S 4$.

An analogue of $r$-inducing in the spin case was provided by Morris and Yaseen [MY] and they called it $(r, \bar{r})$-inducing. To obtain a set of labels which fits the purpose of this procedure we define for $p$ odd inductively a class $\mathscr{C}_{p}(n)$ of partitions behaving well with respect to $(r, \bar{r})$-inducing:

Set $\mathscr{C}_{p}(1)=\{(1)\}$. Assume that the set $\mathscr{C}_{p}(n-1)$ is already defined. If

$$
\lambda=\left(l_{1}, l_{2}, \ldots, l_{m}\right) \in \mathscr{C}_{p}(n-1) \text {, }
$$

where $l_{m}>0$, then

$$
\tilde{\lambda}=\left(l_{1}, l_{2}, \ldots, l_{i-1}, l_{i}+1, l_{i+1}, \ldots, l_{m}\right) \in \mathscr{C}_{p}(n)
$$

if the following two conditions are satisfied:

(i) $l_{i}+1<l_{i-1}$ if $i>1$;

(ii) for $1 \leq j \leq i-1, l_{j}+1 \geq l_{j-1}$ or $\delta_{j} \neq \delta_{i}$ (where $0 \leq \delta_{k}<p / 2$ satisfies $\delta_{k} \equiv l_{k}+(p+1) / 2(\bmod p)$ or $\left.\delta_{k} \equiv-\left(l_{k}+(p+1) / 2\right)(\bmod p)\right)$.

Furthermore, also

$$
\tilde{\lambda}=\left(l_{1}, \ldots, l_{m}, 1\right) \in \mathscr{C}_{p}(n)
$$

if the following two conditions are satisfied:

(i) $1<l_{m}$;

(ii) for $1 \leq j \leq m, l_{j}+1 \geq l_{j-1}$ or $\delta_{j} \neq(p-1) / 2$.

We are then faced with the following problems:

(I) Find an internal description of $\mathscr{C}_{p}(n)$, i.e., find a class $\mathscr{D}_{p}(n)$ of partitions, defined in terms of difference and divisibility conditions on its parts, such that

$$
\mathscr{C}_{p}(n)=\mathscr{D}_{p}(n) \text { for all } n
$$

(II) In view of Brauer's theorem, investigate whether $\left|\mathscr{D}_{p}(n)\right|$ equals the number of partitions of $n$ into distinct parts prime to $p$.

If these problems have a positive answer we have a well-behaved class $\mathscr{D}_{p}(n)$ of labels at hand. For $p=3$ it was found in [BMO] that

$$
\begin{aligned}
\mathscr{D}_{3}(n)=\{ & \left\{\lambda=\left(l_{1}, \ldots, l_{m}\right) \vdash n: \text { for } 1 \leq i \leq m-1,\right. \\
& \left.l_{i}-l_{i+1} \geq 3 \text { and } l_{i}-l_{i+1}>3 \text { if } l_{i} \equiv 0(\bmod 3)\right\} .
\end{aligned}
$$

Thus Problem (II) in this case is settled as a special case of a theorem of Schur [S2, Satz V].

For $p=5$ experimental evidence led to the following conjecture, which was stated in [BMO]:

Conjecture A. $\mathscr{C}_{5}(n)=\mathscr{D}_{5}(n)$, where $\mathscr{D}_{5}(n)=\left\{\lambda=\left(l_{1}, l_{2}, \ldots, l_{m}\right) \vdash n: l_{i}>\right.$ $l_{i+1}$ for all $i \leq m-1 ; l_{i}-l_{i+2} \geq 5$ for all $i \leq m-2 ; l_{i}-l_{i+2}>5$ if $l_{i} \equiv 0$ $(\bmod 5)$ or if $l_{i}+l_{i+1} \equiv 0(\bmod 5)$ for all $i \leq m-2$; there are no subsequences of the following types (for some $j \geq 0):(5 j+3,5 j+2),(5 j+6,5 j+4,5 j)$, $(5 j+5,5 j+1,5 j-1),(5 j+6,5 j+5,5 j, 5 j-1)\}$.

For illustration, we list the partitions contained in $\mathscr{D}_{5}(15)$ :

$(15),(14,1),(13,2),(12,3),(12,2,1),(11,4),(11,3,1),(10,5)$, $(10,4,1),(9,6),(9,5,1),(9,4,2),(8,6,1),(8,5,2),(8,4,3)$, $(7,6,2)$.

It was also conjectured, that problem (II) has a positive answer in this case. 
Conjecture B * $\left|\mathscr{D}_{5}(n)\right|=\left|\mathscr{P}_{5}(n)\right|$, where $\mathscr{P}_{5}(n)$ is the set of partitions of $n$ into distinct parts prime to 5 .

It should at this point be mentioned that for $p=7$ the class $\mathscr{C}_{7}(n)$ does not provide enough labels for the modular characters of $\widehat{S}_{n}$ in characteristic 7. Indeed, for $n=21,\left|\mathscr{C}_{7}(21)\right|=52$, but there are 53 partitions of 21 into distinct parts divisible by 7 . Similar difficulties have been found for $p=11$ and $p=13$ for partitions of $3 p$. An internal description of $\mathscr{C}_{p}(n)$ for $p>5$ similar to the one given above for $p=3$ and $p=5$ will probably be very involved. The experimental evidence shows that there is a difference condition of the form $l_{i}-l_{i+(p-1) / 2} \geq p$ for $\lambda=\left(l_{1}, \ldots, l_{m}\right) \in \mathscr{C}_{p}(n)$, with strict inequality for $l_{i} \equiv 0(\bmod p)$ and in some further cases, and again there is a list of forbidden subsequences. Though we could prove some of these properties even for general $p$, we do not as yet have an internal characterization even for $\mathscr{C}_{7}(n)$.

There is also a refinement of Conjecture $B^{*}$, which is suggested by the representation theoretical context:

If $\lambda=\left(l_{1}, l_{2}, \ldots, l_{m}\right)$ is any partition of $n$ we may define

$$
\begin{aligned}
& a_{\lambda}=\mid\left\{l_{i}\left|l_{i} \equiv 4(\bmod 5)\right|-\left|\left\{l_{i} \mid l_{i} \equiv 1(\bmod 5)\right\}\right|,\right. \\
& b_{\lambda}=\mid\left\{l_{i}\left|l_{i} \equiv 3(\bmod 5)\right|-\left|\left\{l_{i} \mid l_{i} \equiv 2(\bmod 5)\right\}\right|,\right.
\end{aligned}
$$

and

$$
w=w_{\lambda}=\frac{1}{10}\left(2 n-5 a_{\lambda}{ }^{2}-5 b_{\lambda}{ }^{2}-3 a_{\lambda}-b_{\lambda}\right),
$$

a nonnegative integer. For $\alpha, \beta \in \mathbb{Z}$ let

$$
\mathscr{P}(\alpha, \beta ; n)=\left\{\lambda \vdash n \mid a_{\lambda}=\alpha, b_{\lambda}=\beta\right\},
$$

We conjecture that for any $\alpha, \beta \in \mathbb{Z}, n \geq 0$,

$$
\left|\mathscr{P}(\alpha, \beta ; n) \cap \mathscr{D}_{5}(n)\right|=\left|\mathscr{P}(\alpha, \beta ; n) \cap \mathscr{P}_{5}(n)\right| \text {. }
$$

It is known that if $\mathscr{P}(\alpha, \beta ; n) \cap \mathscr{P}_{5}(n) \neq \varnothing$ then with $w$ as above

$$
\left|\mathscr{P}(\alpha, \beta ; n) \cap \mathscr{P}_{5}(n)\right|=\mid\left\{\left(\lambda_{1}, \lambda_{2}\right) \mid \lambda_{1}, \lambda_{2} \text { partitions with }\left|\lambda_{1}\right|+\left|\lambda_{2}\right|=w\right\} \mid
$$

We proceed to provide some background for Conjecture $B$.

The memoir [A3] is devoted to a three parameter extension of the RogersRamanujan identities. The relevant partition functions are as follows.

Definition 1.1. If $l$ is an even integer we denote by $\mathscr{A}_{l, k, a}(n)$ the set of partitions of $n$ satisfying the following conditions:

(i) only multiples of $l+1$ may be repeated,

(ii) no part is $\equiv 0, \pm(a-l / 2)(l+1)(\bmod (2 k-l+1)(l+1))$.

If $l$ is an odd integer we denote by $\mathscr{A}_{l, k, a}(n)$ the set of partitions of $n$ satisfying the following conditions:

(i) only multiples of $(l+1) / 2$ may be repeated,

(ii) no part is $\equiv l+1(\bmod 2 l+2)$,

(iii) no part is $\equiv 0, \pm(2 a-l)(l+1) / 2(\bmod (2 k-l+1)(l+1))$.

We then set $A_{l, k, a}(n)=\left|\mathscr{A}_{l, k, a}(n)\right|$.

Definition 1.2. Let $\mathscr{B}_{l, k, a}(n)$ denote the set of partitions $\lambda=\left(b_{1}, \ldots, b_{s}\right)$ of $n$ satisfying the following conditions:

(i) only multiples of $l+1$ may be repeated, 
(ii) $b_{j}-b_{j+k-1} \geq l+1$ with strict inequality if $b_{j}$ is a multiple of $l+1$,

(iii) denoting the number of appearances of $j$ in our partition by $f_{j}$, then $\sum_{i=j}^{l-j+1} f_{i} \leq a-j$ for $1 \leq j \leq(l+1) / 2$,

(iv) $f_{1}+f_{2}+\cdots+f_{l+1} \leq a-1$.

We then set $B_{l, k, a}(n)=\left|\mathscr{B}_{l, k, a}(n)\right|$.

The main result in $[\mathrm{A} 3]$ is

Theorem [A3, Theorem 8.3]. Let $l, k$ and $a$ be integers with $0 \leq l / 2<a \leq k$ and $l \leq k$; then for each $n \geq 0$

$$
A_{l, k, a}(n)=B_{l, k, a}(n) .
$$

An extensive account is provided in [A3, p.1] and [A1, pp. 205-206] of the classical specializations of this theorem. Suffice it to say here that the celebrated Rogers-Ramanujan identities are the cases $l=0, k=2, a=1,2$.

At the conclusion of [A3, pp. 83-85] it is pointed out that the above result is in certain ways best possible. In particular, the conclusion appears to fall apart if $k<l$. As evidence for this assertion, it is noted that Schur [S2] proves that

$$
A_{3,2,2}(n)=B_{3,2,2}^{0}(n) \text {, }
$$

where $B_{3,2,2}^{0}(n)$ is the number of partitions enumerated by $B_{3,2,2}(n)$ with the added condition that no parts are $\equiv 2(\bmod 4)$. Note that this is just a mild tightening of Definition 1.2 in that condition (iii) in this instance requires that 2 does not appear as a part; the new condition excludes 2 and all other integers $\equiv 2(\bmod 4)$.

The paper [A3] concludes with the next natural case where $k<l$, namely one of the main results to be proved here:

Conjecture B [A3, p. 84, Conjecture 2].

$$
A_{4,3,3}(n)=B_{4,3,3}^{0}(n),
$$

where $B_{4,3,3}^{0}(n)$ denotes the number of partitions of $n$ satisfying the four conditions of Definition 1.2 (with $l=4, k=a=3$ ), and additionally

(v) $f_{5 j+2}+f_{5 j+3} \leq 1$ for $j \geq 0$,

(vi) $f_{5 j+4}+f_{5 j+6} \leq 1$ for $j \geq 0$,

(vii) $f_{5 j-1}+f_{5 j}+f_{5 j+5}+f_{5 j+6} \leq 3$ for $j \geq 1$.

We denote the corresponding set of partitions by $\mathscr{B}_{4,3,3}^{0}(n)$.

We shall prove this conjecture as our Theorem 3.5. Also we point out that when Definition 1.1 is specialized to the case $l=4, k=a=3, \mathscr{A}_{4,3}, 3(n)=$ $\mathscr{P}_{5}(n)$ for all $n$. Moreover, as pointed out in [BMO], there is a bijection between the set $\mathscr{B}_{4,3,3}^{0}(n)=\left\{\lambda=\left(l_{1}, l_{2}, \ldots, l_{m}\right) \vdash n \mid l_{i}>l_{i+1}\right.$ or $l_{i} \equiv 0$ $(\bmod 5)$ for all $i \leq m-1 ; l_{i}-l_{i+2} \geq 5$ for all $i \leq m-2 ; l_{i}-l_{i+2}>5$ if $l_{i} \equiv 0$ (mod 5 ) for all $i \leq m-2$; there are no subsequences of the following types (for some $j \geq 0):(5 j+3,5 j+2),(5 j+6,5 j+4),(5 j+6,5 j+5,5 j, 5 j-1)\}$ and the set $\mathscr{D}_{5}(n)$ defined above: Remove any subsequences $(5 j, 5 j)$ occuring in $\lambda \in \mathscr{B}_{4,3,3}^{0}(n)$ and replace them by $5 j+1,5 j-1$. We therefore have that Conjecture $B$ and Conjecture $B^{*}$ are equivalent.

Conjecture B was tested for $n \leq 59$ and found to be correct [A3]. As remarked in the next to last paragraphs of [A3, p. 85], "Unfortunately the assumption $k \geq l$ so permeates the work in this paper that Conjecture 2 seems well 
beyond the techniques herein introduced". Nothing that follows contradicts this assertion; the methods we shall follow are based upon the ideas in [A2, A5, and A4, $\S 10.6]$ and are quite unlike those of [A3].

We shall, in fact, prove a refinement of the above conjecture. To this end we make two special definitions.

Definition 1.3. We denote by $A(\mu, \nu ; N)$ the number of partitions of $N$ into distinct nonmultiples of 5 of which $\mu$ are congruent to 1 or $2(\bmod 5)$ and $\nu$ are congruent to 3 or $4(\bmod 5)$.

Definition 1.4. We denote by $B(\mu, \nu ; N)$ the number of partitions $\lambda=\left(b_{1}, \ldots\right.$, $b_{s}$ ) of $N$ satisfying the following conditions:

(i) only multiples of 5 may be repeated,

(ii) $b_{j}-b_{j+2} \geq 5$ with strict inequality if $b_{j}$ is a multiple of 5 ,

(iii) denoting the number of appearances of $j$ in our partition by $f_{j}$, we require

$$
\begin{aligned}
f_{5 j+2}+f_{5 j+3} & \leq 1, & & \text { for } j \geq 0, \\
f_{5 j+4}+f_{5 j+6} & \leq 1, & & \text { for } j \geq 0, \\
f_{5 j-1}+f_{5 j}+f_{5 j+5}+f_{5 j+6} & \leq 3, & & \text { for } j \geq 1,
\end{aligned}
$$

(iv) there are $\mu$ parts of the partition $\equiv 0,1$ or $2(\bmod 5)$,

(v) there are $\nu$ parts of the partition $\equiv 0,3$ or $4(\bmod 5)$.

In $\S 3$ we prove

Theorem 3.1. For each $\mu, \nu, N \geq 0$,

$$
A(\mu, \nu ; N)=B(\mu, \nu ; N) \text {. }
$$

For example, $A(2,2 ; 15)=4$, the relevant partitions being $9+3+2+1$, $8+4+2+1,7+4+3+1$, and $6+4+3+2$; while $B(2,2 ; 15)=4$ also, the relevant partitions being $10+5,10+4+1,9+5+1,8+5+2$.

The next section is devoted to the study of recurrences for polynomial generating functions arising from Definition 1.4.

\section{THE RECURRENCES}

We begin by noting that for any partition of any integer which satisfies (i)(iii) of Definition 1.4 there are exactly 16 possibilities (numbered $0-15$ ) for the subset of summands of the partition that lie in the interval $[5 j+1,5 j+5]$, namely

$0: \varnothing \quad$ (the empty set)

1: $5 j+1$

2: $5 j+2$

3: $5 j+2,5 j+1$

4: $5 j+3$

5: $5 j+3,5 j+1$

6: $5 j+4$

7: $5 j+4,5 j+1$

8: $5 j+4,5 j+2$

9: $5 j+4,5 j+3$

10: $5 j+5$ 

11: $5 j+5,5 j+1$
12: $5 j+5,5 j+2$
13: $5 j+5,5 j+3$
14: $5 j+5,5 j+4$
15: $5 j+5,5 j+5$.

We now place an ordering on these 16 sets by ordering them lexicographically from left to right (i.e., the list is presented in increasing order).

Definition 2.1. We define $S_{n}(j ; a, b ; q)$ to be the generating function for all partitions satisfying (i)-(iii) of Definition 1.4 and in addition: (vi) all parts are $\leq 5 n+5$, (vii) the subset of summands that lie in the interval [ $5 n+1,5 n+5]$ must have number $\leq j$ on the above list. The exponent on $q$ is the number being partitioned. The exponent on $a$ is the number of summands congruent to 0,1 or $2(\bmod 5)$, and the exponent on $b$ is the number of summands congruent to 0,3 or $4(\bmod 5)$. When $n=-1$, we define $S_{-1}(j ; a, b ; q)=$ 1 , and when $n<-1$, we define $S_{n}(j ; a, b ; q)=0$.

For example,

$$
\begin{aligned}
S_{0}(9 ; a, b, q)= & 1+a q+a q^{2}+a^{2} q^{3}+b q^{3}+a b q^{4} \\
& +b q^{4}+a b q^{5}+a b q^{6}+b^{2} q^{7}
\end{aligned}
$$

and

$$
\begin{aligned}
S_{0}(15 ; a, b ; q)= & 1+a q+a q^{2}+a^{2} q^{3}+b q^{3}+a b q^{4}+b q^{4}+2 a b q^{5}+a b q^{6} \\
& +a^{2} b q^{6}+a^{2} b q^{7}+b^{2} q^{7}+a b^{2} q^{8}+a b^{2} q^{9}+a^{2} b^{2} q^{10}
\end{aligned}
$$

Surprisingly (although less so after seeing $\S 3$ ),

$$
S_{0}(15 ; a, b ; q)=(1+a q)\left(1+a q^{2}\right)\left(1+b q^{3}\right)\left(1+b q^{4}\right)
$$

It is now a fairly routine matter to state the 16 defining recurrence relations for $S_{n}(j ; a, b ; q)$.

To simplify the statements we write $S_{n}(j)$ for $S_{n}(j ; a, b ; q)$ throughout. Now for each $n \geq 0$,

$$
\begin{aligned}
S_{n}(0)= & S_{n-1}(15) \\
S_{n}(1)= & S_{n}(0)+a q^{5 n+1}\left(S_{n-1}(11)-S_{n-1}(9)+S_{n-1}(5)\right) \\
& -a^{3} b^{3} q^{20 n-10} S_{n-3}(9) \\
S_{n}(2)= & S_{n}(1)+a q^{5 n+2}\left(S_{n-1}(12)-S_{n-1}(9)+S_{n-1}(8)\right) \\
S_{n}(3)= & S_{n}(2)+a^{2} q^{10 n+3} S_{n-1}(3) \\
S_{n}(4)= & S_{n}(3)+b q^{5 n+3} S_{n-1}(13) \\
S_{n}(5)= & S_{n}(4)+a b q^{10 n+4} S_{n-1}(5) \\
S_{n}(6)= & S_{n}(5)+b q^{5 n+4} S_{n-1}(14)
\end{aligned}
$$




$$
\begin{aligned}
S_{n}(7) & =S_{n}(6)+a b q^{10 n+5} S_{n-1}(5) \\
S_{n}(8) & =S_{n}(7)+a b q^{10 n+6} S_{n-1}(8) \\
S_{n}(9) & =S_{n}(8)+b^{2} q^{10 n+7} S_{n-1}(9) \\
S_{n}(10) & =S_{n}(9)+a b q^{5 n+5} S_{n-1}(14) \\
S_{n}(11) & =S_{n}(10)+a^{2} b q^{10 n+6} S_{n-1}(5) \\
S_{n}(12) & =S_{n}(11)+a^{2} b q^{10 n+7} S_{n-1}(8) \\
S_{n}(13) & =S_{n}(12)+a b^{2} q^{10 n+8} S_{n-1}(9) \\
S_{n}(14) & =S_{n}(13)+a b^{2} q^{10 n+9} S_{n-1}(9) \\
S_{n}(15) & =S_{n}(14)+a^{2} b^{2} q^{10 n+10} S_{n-1}(9)
\end{aligned}
$$

We now describe how these 16 recurrences are proved. First we consider general observations common to all 16; then we shall carry out the details in a few cases. The remainder will follow in a similar manner.

In each of these recurrences, we see for $j>0$

$$
S_{n}(j)=S_{n}(j-1)+\cdots .
$$

Now $S_{n}(j)-S_{n}(j-1)$ is the generating function for all those admissible partitions with precisely the $j$ th subset of summands in the interval [ $5 n+1,5 n+5]$.

As a prototypical example, let us consider (2.15). Thus $S_{n}(15)-S_{n}(14)$ must generate partitions whose largest summands are $5 n+5$ taken twice. Once we know that $b_{1}=b_{2}=5 n+5$, we see immediately that $b_{3} \leq 5 n-1$, and we see on a moments reflection that these partitions are generated by $a b q^{5 n+5} a b q^{5 n+5} S_{n-1}(9)$. Hence

$$
S_{n}(15)-S_{n}(14)=a^{2} b^{2} q^{10 n+10} S_{n-1}(9),
$$

which is $(2.15)$.

The only real exception to the above pattern is $(2.0)$. Clearly $S_{n}(0)$ generates all admissible partitions with largest part $\leq 5 n$. Hence we see (2.0) immediately, namely $S_{n}(0)=S_{n-1}(15)$.

The most intricate of these recurrences is (2.1). As above we see that $S_{n}(1)-$ $S_{n}(0)$ generates those admissible partitions whose only summand in $[5 n+$ $1,5 n+5]$ is precisely $5 n+1$. Which of the 16 possible subsets can be allowed in $[5 n-4,5 n]$ ? Examination reveals the numbers $0,1,2,3,4,5,10$, and 11. Thus the admissible partitions are generated by

$$
\left.a q^{5 n+1}\left(S_{n-1}(11)\right)-S_{n-1}(9)+S_{n-1}(5)\right) .
$$

However this is not quite correct in that the above allows partitions whose top four parts are $5 n+1,5 n, 5 n-5,5 n-6$. Hence we must subtract off

$$
a q^{5 n+1} a b q^{5 n} a b q^{5 n-5} b q^{5 n-6} S_{n-3}(9)=a^{3} b^{3} q^{20 n-10} S_{n-3}(9) \text {. }
$$

Therefore

$S_{n}(1)-S_{n}(0)=a q^{5 n+1}\left(S_{n-1}(11)-S_{n-1}(9)+S_{n-1}(5)\right)-a^{3} b^{3} q^{20 n-10} S_{n-3}(9)$,

which is effectively (2.1).

The remaining 13 formulas are proved in a similar manner. 
We now define two important linear combinations of the sequences $S_{n}(9)$ and $S_{n}(15)$. Namely

$$
\begin{aligned}
J(n)= & S_{n}(9)-\left(1-q^{5 n}\right)\left(1+a q^{5 n+1}+a q^{5 n+2}+b q^{5 n+3}+b q^{5 n+4}\right) S_{n-1}(15) \\
& -q^{5 n}\left(1+a q^{5 n+1}+a q^{5 n+2}+a^{2} q^{5 n+3}+b q^{5 n+3}+b q^{5 n+4}\right. \\
& \left.+a b q^{5 n+4}+a b q^{5 n+5}+a b q^{5 n+6}+b^{2} q^{5 n+7}\right) S_{n-1}(9) \\
& +\left(1-q^{5 n}\right) a b q^{15 n-2}\left(a^{2}+a b q+a b q^{2}+a b q^{3}+a^{2} b q^{3}\right. \\
& \left.+a^{2} b q^{4}+b^{2} q^{4}+a b^{2} q^{5}+a b^{2} q^{6}\right) S_{n-2}(9) \\
& +a^{3} b^{3} q^{20 n-10}\left(1-q^{5 n}\right)\left(1-q^{5 n-5}\right) S_{n-3}(9)
\end{aligned}
$$

and

$$
\begin{aligned}
K(n)= & S_{n}(9)-S_{n}(15)+a b q^{5 n+5}\left(1-q^{5 n}\right) S_{n-1}(15) \\
& +a b q^{10 n+5}\left(1+a q+a q^{2}+b q^{3}+b q^{4}+a b q^{5}\right) S_{n-1}(9) \\
& -a^{3} b^{3} q^{15 n+5}\left(1-q^{5 n}\right) S_{n-2}(9) .
\end{aligned}
$$

Lemma 2.2. For $n \geq 0$

$$
J(n)=K(n)=0 .
$$

Proof. This result is easily obtained from (2.0)-(2.15). First we note that the other 14 sequences $S_{n}(j) \quad(j \neq 9,15)$ may be defined as combinations of $S_{n}(9)$ and $S_{n}(15)$ as follows. Equation (2.15) yields $S_{n}(14)$ as such a combination (namely $S_{n}(15)-a^{2} b^{2} q^{10 n+10} S_{n-1}(9)$ ). Equations (2.14) and (2.13) in that order then yield $S_{n}(13)$ and $S_{n}(12)$. Equation (2.9) yields $S_{n}(8)$. Then (2.12) yields $S_{n}(11)$, and (2.10) yields $S_{n}(10)$. Now (2.11) (with $n$ replaced by $n+1)$ yields

$$
S_{n}(5)=a^{-2} b^{-1} q^{-10 n-16}\left(S_{n+1}(11)-S_{n+1}(10)\right),
$$

which in turn yields $S_{n}(5)$ in terms of $S_{n}(9)$ and $S_{n}(15)$. Equation (2.8) yields $S_{n}(7)$, and (2.7) yields $S_{n}(6)$. Equations (2.5), (2.4), (2.3) and (2.2) in that order yield $S_{n}(4), S_{n}(3), S_{n}(2)$, and $S_{n}(1)$. Finally (2.0) yields $S_{n}(0)$.

Substituting the expressions for $S_{n}(6), S_{n}(5)$, and $S_{n-1}(14)$ into (2.6) proves that $K(n)=0$ for $n \geq 0$. Finally substituting the expressions we have obtained for $S_{n}(1), S_{n}(0), S_{n-1}(11)$, and $S_{n-1}(5)$ into (2.1) yields

$$
0=a^{2} b q^{10 n+16} J(n)-K(n+1)+a q^{5 n+8}\left(q^{3}+a q^{5 n+5}+b q^{5 n+6}+b q^{5 n+7}\right) K(n) .
$$

We have already seen that $K(n)$ is zero for each $n \geq 0$; hence $J(n)=0$, i.e., (2.16) is valid.

\section{Proof of Conjecture B}

Theorem 3.1. For all $\mu, \nu, n \geq 0 A(\mu, \nu ; n)=B(\mu, \nu ; n)$.

The engine for proving Theorem 3.1 is Lemma 3.4, which gives a surprisingly simple functional equation relating $S_{n}(15 ; a, b ; q)$ to $S_{n-1}\left(9 ; a q^{5}, b q^{5} ; q\right)$. Once this result is established, the main result follows easily. 
Lemma 3.2. For $n \geq 0$,

$$
\begin{aligned}
&\left(1+a q^{5 n-4}+a q^{5 n-3}+b q^{5 n-2}+b q^{5 n-1}\right) S_{n}(9) \\
&= p_{1}(n ; a, b ; q) S_{n-1}(9)+p_{2}(n ; a, b ; q) S_{n-2}(9)\left(1-q^{5 n}\right) \\
&+p_{3}(n ; a, b ; q)\left(1-q^{5 n}\right)\left(1-q^{5 n-5}\right) S_{n-3}(9) \\
&+a^{4} b^{4} q^{25 n-30}\left(1+a q^{5 n+1}+a q^{5 n+2}+b q^{5 n+3}+b q^{5 n+4}\right) \\
& \cdot\left(1-q^{5 n}\right)\left(1-q^{5 n-5}\right)\left(1-q^{5 n-10}\right) S_{n-4}(9),
\end{aligned}
$$

where

$$
\begin{aligned}
p_{1}(n ; a, b ; q)= & b^{3} q^{15 n+6}+b^{3} q^{15 n+5}+a b^{2} q^{15 n+5}+2 a b^{2} q^{15 n+4}+2 a b^{2} q^{15 n+3} \\
& +a^{2} b q^{15 n+3}+a b^{2} q^{15 n+2}+2 a^{2} b q^{15 n+2}+2 a^{2} b q^{15 n+1} \\
& +a^{3} q^{15 n-1}+b^{2} q^{10 n+7}+a b q^{10 n+6}+a b q^{10 n+5}+a b^{2} q^{10 n+4} \\
& +a b q^{10 n+4}+a b^{2} q^{10 n+3}+b^{2} q^{10 n+3}+a^{2} q^{10 n+3}+2 b^{2} q^{10 n+2} \\
& +a^{2} b q^{10 n+2}+b^{2} q^{10 n+1}+a^{2} b q^{10 n+1}+2 a b q^{10 n+1}+2 a b q^{10 n-1} \\
& +a^{2} q^{10 n-1}+2 a^{2} q^{10 n-2}+a^{2} q^{10 n-3}+b q^{5 n+4}+b q^{5 n+3} \\
& +a q^{5 n+2}+a q^{5 n+1}+b q^{5 n-1}+b q^{5 n-2}+a q^{5 n-3}+a q^{5 n-4} \\
& +a^{2} b q^{15 n}+a^{3} q^{15 n}+3 a b q^{10 n}+a b q^{5 n}+1
\end{aligned}
$$

$$
\begin{aligned}
p_{2}(n ; a, b ; q)= & a b^{4} q^{20 n+2}+a b^{4} q^{20 n+1}+a b^{4} q^{20 n-1}+3 a^{2} b^{3} q^{20 n-1} \\
& +3 a^{2} b^{3} q^{20 n-2}+a^{3} b^{2} q^{20 n-2}+a^{2} b^{3} q^{20 n-3}+3 a^{3} b^{2} q^{20 n-3} \\
& +3 a^{3} b^{2} q^{20 n-4}+a^{4} b q^{20 n-4}+a^{3} b^{2} q^{20 n-5}+a^{4} b q^{20 n-5} \\
& +a^{4} b q^{20 n-6}+a^{4} b q^{20 n-7}+a b^{3} q^{15 n+3}+a b^{3} q^{15 n+2} \\
& +a b^{3} q^{15 n+1}+a^{2} b^{2} q^{15 n+1}+a^{2} b^{3} q^{15 n-1}+a^{2} b^{2} q^{15 n-1} \\
& +a^{3} b q^{15 n-1}+a^{2} b^{3} q^{15 n-2}+a b^{3} q^{15 n-2}+a^{3} b q^{15 n-2} \\
& +a b^{3} q^{15 n-3}+a^{3} b^{2} q^{15 n-3}+a^{3} b q^{15 n-3}+a b^{3} q^{15 n-4} \\
& +a^{3} b^{2} q^{15 n-4}+a^{2} b^{2} q^{15 n-4}+3 a^{2} b^{2} q^{15 n-5}+a^{2} b^{2} q^{15 n-6} \\
& +a^{3} b q^{15 n-6}+a^{3} b q^{15 n-7}+a^{3} b q^{15 n-8}+a b^{2} q^{10 n-1} \\
& +a b^{2} q^{10 n-2}+a^{2} b q^{10 n-3}+a^{2} b q^{10 n-4}+a b^{4} q^{20 n} \\
& +a^{2} b^{3} q^{20 n}+3 a^{2} b^{2} q^{15 n}+a^{2} b^{2} q^{10 n}
\end{aligned}
$$

and

$$
\begin{aligned}
p_{3}(n ; a, b ; q)= & a^{2} b^{5} q^{25 n-9}+a^{2} b^{5} q^{25 n-10}+a^{3} b^{4} q^{25 n-10} \\
& +2 a^{3} b^{4} q^{25 n-11}+2 a^{3} b^{4} q^{25 n-12}+a^{4} b^{3} q^{25 n-12}+a^{3} b^{4} q^{25 n-13} \\
& +2 a^{4} b^{3} q^{25 n-13}+2 a^{4} b^{3} q^{25 n-14}+a^{4} b^{3} q^{25 n-15}+a^{5} b^{2} q^{25 n-15} \\
& +a^{5} b^{2} q^{25 n-16}-a^{3} b^{4} q^{20 n-6}-a^{3} b^{4} q^{20 n-7}-a^{4} b^{3} q^{20 n-8} \\
& -a^{4} b^{3} q^{20 n-9}-a^{3} b^{3} q^{20 n-10}+a^{2} b^{4} q^{20 n-13}+a^{3} b^{3} q^{20 n-14} \\
& +a^{3} b^{3} q^{20 n-15}+a^{3} b^{3} q^{20 n-16}+a^{4} b^{2} q^{20 n-17}-a^{3} b^{3} q^{15 n-10}
\end{aligned}
$$

Proof. While the exact expressions for $p_{1}(n ; a, b ; q), p_{2}(n ; a, b ; q)$ and $p_{3}(n ; a, b ; q)$ are onerous, the proof of $(3.1)$ is quite routine. Comparing 
(2.18) with (2.16), we see that $S_{n-1}(15)$ may be written as a linear combination of $S_{n}(9), S_{n-1}(9), S_{n-2}(9)$ and $S_{n-3}(9)$. Noting from (2.18) that $K(n)=0$, we substitute for each appearance of $S_{n}(15)$ and $S_{n-1}(15)$ in (2.17) our new combination of $S_{n-i}(9)$. The result is (3.1) after simplification and the replacement of $n$ by $n-1$.

Lemma 3.3. For $n \geq 0$,

$$
\begin{aligned}
\left(1+a q^{5 n-4}+a q^{5 n-3}+b q^{5 n-2}+b q^{5 n-1}\right) S_{n}(15) \\
\quad=p_{1}\left(n-1 ; a q^{5}, b q^{5} ; q\right) S_{n-1}(15) \\
\quad+p_{2}\left(n-1 ; a q^{5}, b q^{5} ; q\right) S_{n-2}(15)\left(1-q^{5 n-5}\right) \\
\quad+p_{3}\left(n-1 ; a q^{5}, b q^{5} ; q\right) S_{n-3}(15)\left(1-q^{5 n-5}\right)\left(1-q^{5 n-10}\right) \\
\quad+a^{4} b^{4} q^{25 n-15}\left(1+a q^{5 n+1}+a q^{5 n+2}+b q^{5 n+3}+b q^{5 n+4}\right) \\
\quad \cdot\left(1-q^{5 n-5}\right)\left(1-q^{5 n-10}\right)\left(1-q^{5 n-15}\right) S_{n-4}(15) .
\end{aligned}
$$

Proof. For $n \geq 0$, we define $\Delta(n)$ to be the left-hand side of (3.5) minus the right-hand side of (3.5) with $n$ replaced by $n+1$. Then

$$
\begin{aligned}
\Delta(n)= & \left(1+a q^{5 n+1}+a q^{5 n+2}+b q^{5 n+3}+b q^{5 n+4}\right) K(n+1) \\
& +p_{4}(n ; a, b ; q) J(n+1)+p_{5}(n ; a, b ; q) K(n) \\
& +p_{6}(n ; a, b ; q) J(n)+p_{7}(n ; a, b ; q) K(n-1) \\
& +p_{8}(n ; a, b ; q) J(n-1)+p_{9}(n ; a, b ; q) K(n-2),
\end{aligned}
$$

where

$$
\begin{aligned}
p_{4}(n ; a, b ; q) & =1+a q^{5 n+1}+a q^{5 n+2}+b q^{5 n+3}+b q^{5 n+4} \\
p_{5}(n ; a, b ; q)= & -b^{3} q^{15 n+21}-b^{3} q^{15 n+20}-a b^{2} q^{15 n+20}-3 a b^{2} q^{15 n+19} \\
& -3 a b^{2} q^{15 n+18}-b^{2} q^{15 n+18}-a^{2} b q^{15 n+18}-a b^{2} q^{15 n+17} \\
& -2 b^{2} q^{15 n+17}-3 a^{2} b q^{15 n+17}-b^{2} q^{15 n+16}-3 a^{2} b q^{15 n+16} \\
& -2 a b q^{15 n+16}-a^{2} b q^{15 n+15}-4 a b q^{15 n+15}-a^{3} q^{15 n+15} \\
& -2 a b q^{15 n+14}-a^{3} q^{15 n+14}-a^{2} q^{15 n+14}-2 a^{2} q^{15 n+13} \\
& -a^{2} q^{15 n+12}-a b^{2} q^{10 n+19}-a b^{2} q^{10 n+18}-b^{2} q^{10 n+17} \\
& -a^{2} b q^{10 n+17}-a^{2} b q^{10 n+16}-a b q^{10 n+16}-2 a b q^{10 n+15} \\
& +a b^{2} q^{10 n+14}-a b q^{10 n+14}-b q^{10 n+14}+a b^{2} q^{10 n+13} \\
& -b q^{10 n+13}-a^{2} q^{10 n+13}+a^{2} b q^{10 n+12}-a q^{10 n+12} \\
& +a^{2} b q^{10 n+11}-a q^{10 n+11}+a b q^{10 n+10}-b q^{10 n+9} \\
& -b q^{10 n+8}-a q^{10 n+7}-a q^{10 n+6}-q^{5 n+5}
\end{aligned}
$$

$$
\begin{aligned}
p_{6}(n ; a, b ; q)= & a^{2} b^{3} q^{15 n+24}+a^{2} b^{3} q^{15 n+23}+a b^{3} q^{15 n+23}+2 a b^{3} q^{15 n+22} \\
& +a^{3} b^{2} q^{15 n+22}+a b^{3} q^{15 n+21}+a^{3} b^{2} q^{15 n+21}+2 a^{2} b^{2} q^{15 n+21} \\
& +4 a^{2} b^{2} q^{15 n+20}+2 a^{2} b^{2} q^{15 n+19}+a b^{2} q^{15 n+19}+a^{3} b q^{15 n+19} \\
& +a b^{2} q^{15 n+18}+2 a^{3} b q^{15 n+18}+a^{3} b q^{15 n+17}+a^{2} b q^{15 n+17} \\
& +a^{2} b q^{15 n+16}+a^{2} b^{2} q^{10 n+20}+a b^{2} q^{10 n+14}+a b^{2} q^{10 n+13} \\
& +a^{2} b q^{10 n+12}+a^{2} b q^{10 n+11}+a b q^{10 n+10}
\end{aligned}
$$


(3.10)

$$
\begin{aligned}
p_{7}(n ; a, b ; q) & \\
=\left(1-q^{5 n}\right) \cdot & \left(a^{2} b^{4} q^{20 n+28}+2 a^{2} b^{4} q^{20 n+27}+a^{2} b^{4} q^{20 n+26}+a b^{4} q^{20 n+26}\right. \\
& +2 a^{3} b^{3} q^{20 n+26}+a b^{4} q^{20 n+25}+4 a^{3} b^{3} q^{20 n+25}+a^{2} b^{3} q^{20 n+25} \\
& +2 a^{3} b^{3} q^{20 n+24}+3 a^{2} b^{3} q^{20 n+24}+a^{4} b^{2} q^{20 n+24}+3 a^{2} b^{3} q^{20 n+23} \\
& +2 a^{4} b^{2} q^{20 n+23}+a^{3} b^{2} q^{20 n+23}+a^{2} b^{3} q^{20 n+22}+a^{4} b^{2} q^{20 n+22} \\
& +3 a^{3} b^{2} q^{20 n+22}+3 a^{3} b^{2} q^{20 n+21}+a^{3} b^{2} q^{20 n+20}+a^{4} b q^{20 n+20} \\
& +a^{4} b q^{20 n+19}-a^{2} b^{2} q^{15 n+20}+a^{2} b^{3} q^{15 n+19}+a^{2} b^{3} q^{15 n+18} \\
& +a b^{3} q^{15 n+17}+a^{3} b^{2} q^{15 n+17}+a^{3} b^{2} q^{15 n+16}+a^{2} b^{2} q^{15 n+16} \\
& \left.+2 a^{2} b^{2} q^{15 n+15}+a^{2} b^{2} q^{15 n+14}+a^{3} b q^{15 n+13}\right)
\end{aligned}
$$

$$
\begin{aligned}
p_{8}(n ; a, b ; q)=-\left(1-q^{5 n}\right)\left(a^{3} b^{4} q^{20 n+29}\right. & +a^{3} b^{4} q^{20 n+28}+a^{4} b^{3} q^{20 n+27} \\
& \left.+a^{4} b^{3} q^{20 n+26}+a^{3} b^{3} q^{15 n+20}\right),
\end{aligned}
$$

$$
\begin{aligned}
p_{9}(n ; a, b ; q) & \\
=\left(1-q^{5 n}\right)\left(1-q^{5 n-5}\right)\left(a^{3} b^{4} q^{25 n+24}\right. & +a^{3} b^{4} q^{25 n+23}+a^{4} b^{3} q^{25 n+22} \\
& \left.+a^{4} b^{3} q^{25 n+21}+a^{3} b^{3} q^{20 n+15}\right) .
\end{aligned}
$$

Identity (3.6) is conceptually quite easy; both sides are just linear combinations of $S_{n-i}(9)$ and $S_{n-i}(15)$ with polynomial coefficients. It is a simple matter for MACSYMA to show that each side is the same combination.

Applying Lemma 2.2 to (3.6), we see that $\Delta(n)=0$ for $n \geq 2$. MACSYMA then may easily verify that $\Delta(0)=\Delta(1)=0$.

Lemma 3.4. For $n \geq 0$

$$
\begin{aligned}
& S_{n}(15 ; a, b ; q) \\
& \quad=(1+a q)\left(1+a q^{2}\right)\left(1+b q^{3}\right)\left(1+b q^{4}\right) S_{n-1}\left(9 ; a q^{5}, b q^{5} ; q\right) .
\end{aligned}
$$

Proof. Comparing Lemma 3.3 and Lemma 3.2, we see that both sides of (3.13) satisfy exactly the same fourth order recurrence valid for $n \geq 1$. Thus we need only check that (3.13) is valid for the initial values of $n=0,1,2,3$, and MACSYMA performs this task without difficulty. Hence (3.13) is valid for each $n \geq 0$.

We are now ready to prove our main result.

Proof of Theorem 3.1. Clearly for $0 \leq j \leq 15$,

$$
\lim _{n \rightarrow \infty} S_{n}(j ; a, b ; q)=\sum_{\mu, \nu, N \geq 0} B(\mu, \nu ; N) a^{\mu} b^{\nu} q^{N} \equiv S(a, b, q) .
$$

Hence letting $n \rightarrow \infty$ in (3.13), we find that

$$
S(a, b, q)=(1+a q)\left(1+a q^{2}\right)\left(1+b q^{3}\right)\left(1+b q^{4}\right) S\left(a q^{5}, b q^{5}, q\right) .
$$


Iterating (3.15), we see that

$$
\begin{aligned}
S(a, b, q) & =\prod_{n=0}^{\infty}\left(1+a q^{5 n+1}\right)\left(1+a q^{5 n+2}\right)\left(1+b q^{5 n+3}\right)\left(1+b q^{5 n+4}\right) \\
& =\sum_{\mu, \nu, N \geq 0} A(\mu, \nu ; N) a^{\mu} b^{\nu} q^{N}
\end{aligned}
$$

Comparing (3.14) with (2.16), we see that

$$
A(\mu, \nu ; N)=B(\mu, \nu, N)
$$

for all nonnegative $\mu, \nu$ and $N$.

Theorem 3.5. Conjecture B is valid.

Proof.

$$
A_{4,3,3}(N)=\sum_{\mu, \nu \geq 0} A(\mu, \nu ; N)=\sum_{\mu, \nu \geq 0} B(\mu, \nu ; N)=B_{4,3,3}^{0}(N) .
$$

\section{Proof of Conjecture A}

This section is devoted to the proof of Conjecture A, i.e.:

Theorem 4.1. For all $n \in \mathbb{N}$, we have

$$
\mathscr{C}_{5}(n)=\mathscr{D}_{5}(n) .
$$

(The sets $\mathscr{C}_{5}(n)$ and $\mathscr{D}_{5}(n)$ were defined in $\S 1$.)

Since the proofs of the two inclusions $\mathscr{C}_{5}(n) \subseteq \mathscr{D}_{5}(n)$ and $\mathscr{D}_{5}(n) \subseteq \mathscr{C}_{5}(n)$ are quite different, we break the proof of the theorem up into these two parts.

Proposition 4.2. For all $n \in \mathbb{N}$, we have $\mathscr{C}_{5}(n) \subseteq \mathscr{D}_{5}(n)$.

Proof. Assume the statement is false, and let $n$ be minimal with $\mathscr{C}_{5}(n) \nsubseteq$ $\mathscr{D}_{5}(n)$. So $n>1$ and we can take $\lambda \in \mathscr{C}_{5}(n), \lambda \notin \mathscr{D}_{5}(n)$.

By the construction rules of $\mathscr{C}_{5}(n), \lambda=\left(l_{1}, \ldots, l_{m}\right)$ where $l_{1}>l_{2}>\ldots>$ $l_{m}$. As $\lambda \notin \mathscr{D}_{5}(n)$, one of the conditions for $\mathscr{D}_{5}$-partitions must be false for $\lambda$.

(i) Suppose $l_{i}-l_{i+2}<5$ for some $i$, and let $i$ be minimal with this. As a predecessor of $\lambda$ is in $\mathscr{C}_{5}(n-1)$ and $\mathscr{C}_{5}(n-1) \subseteq \mathscr{D}_{5}(n-1)$, a predecessor of $\lambda$ must be of the form

$$
\tilde{\lambda}: l_{1}>\cdots>l_{i}>l_{i+1}>l_{i+2}-1>l_{i+3}>\cdots>l_{m}
$$

and $l_{i}-\left(l_{i+2}-1\right) \geq 5$, hence $l_{i+2}-1=l_{i}-5$, or $l_{i+2}=1$ and $\tilde{\lambda}: l_{1}>\cdots>$ $l_{i}>l_{i+1}$ with $l_{i}=4$.

In both cases, $l_{i} \neq l_{i-1}-1$ as otherwise $l_{i-1}-l_{i+1}<5$, contradicting the minimality of $i$. In the first case, $\delta\left(l_{i}\right)=\delta\left(l_{i}-5\right)=\delta\left(l_{i+2}-1\right)$ and hence $\tilde{l}_{i+2}=l_{i+2}-1$ cannot be increased to obtain $\lambda$ from $\tilde{\lambda}$. In the second case, $\delta\left(l_{i}\right)=\delta(4)=2=\delta(0)$, and hence also here $\lambda$ cannot be obtained from $\tilde{\lambda}$. Thus there would be no predecessor of $\lambda$, a contradiction.

(ii) Suppose $l_{i}-l_{i+2}=5$ and $l_{i} \equiv 0$ for some $i$, and let $i$ be minimal with this. The only possible predecessor for $\lambda$ in $\mathscr{C}_{5}(n-1) \subseteq \mathscr{D}_{5}(n-1)$ is

$$
\tilde{\lambda}: l_{1}>\cdots>l_{i}>l_{i+1}>l_{i+2}-1>\cdots>l_{m} \text {. }
$$


Now $\delta\left(l_{i}\right)=\delta(0)=2=\delta(4)=\delta\left(l_{i+2}-1\right)$, so if we are allowed to increase $\tilde{l}_{i+2}=l_{i+2}-1$, then we must have $l_{i-1}=l_{i}+1$. But then $l_{i+1}=l_{i-1}-5$ as $l_{i-1}-l_{i+1}=l_{i}+1-l_{i+1} \geq 5$ and $l_{i}-l_{i+1}<l_{i}-l_{i+2}=5$, so $\tilde{\lambda}$ contains a forbidden subsequence $\left(l_{i}, l_{i+1}, \tilde{l}_{i+2}\right)=(5 k, 5 k-4,5 k-6)$, a contradiction.

(iii) Suppose $\lambda$ has a subsequence $\left(l_{i}, l_{i+1}\right)=(5 k+3,5 k+2)$ for some $i$, and let $i$ be minimal with this. A predecessor can only be

$$
\tilde{\lambda}: l_{1}>\cdots>l_{i}>l_{i+1}-1>\cdots \text {. }
$$

Now $l_{i-1}-\left(l_{i+1}-1\right) \geq 5$ implies $l_{i-1}-l_{i}=l_{i-1}-\left(l_{i+1}+1\right) \geq 3$, and $\delta\left(l_{i}\right)=$ $\delta(3)=1=\delta(1)=\delta\left(l_{i+1}-1\right)$, so $\tilde{l}_{i+1}=l_{i+1}-1$ cannot be raised, a contradiction.

(iv) Suppose $l_{i}-l_{i+2}=5$ and $l_{i}+l_{i+1} \equiv 0(\bmod 5)$ for some $i$, and let $i$ be minimal with this. By (iii), $\left(l_{i}, l_{i+1}\right) \neq(5 k+3,5 k+2)$ for some $k$, so we must have $\left(l_{i}, l_{i+1}\right)=(5 k+4,5 k+1)$ or $(5 k+1,5 k-1)$ for some $k$.

Consider first the case $\left(l_{i}, l_{i+1}\right)=(5 k+4,5 k+1)$. Note that $l_{i-1}-l_{i} \geq 2$, since by (i) $l_{i-1}-l_{i+1} \geq 5$. Possible predecessors for $\lambda$ are (a) $\tilde{\lambda}: \cdots l_{i}>$ $l_{i+1}-1>l_{i+2}>\ldots$ or (b) $\tilde{\lambda}: \cdots l_{i}>l_{i+1}>l_{i+2}-1>\cdots$. But

$$
\delta\left(l_{i+1}-1\right)=\delta(0)=2=\delta(4)=\delta\left(l_{i}\right)
$$

and

$$
\delta\left(l_{i+2}-1\right)=\delta(3)=1=\delta(1)=\delta\left(l_{i+1}\right),
$$

and hence in both cases we cannot construct $\lambda$ from $\tilde{\lambda}$, a contradiction.

Now consider the second case $\left(l_{i}, l_{i+1}\right)=(5 k+1,5 k-1)$. Possible predecessors are (a) $\tilde{\lambda}: \cdots l_{i}>l_{i+1}-1>l_{i+2}>\cdots$ or (b) $\tilde{\lambda}: \cdots l_{i}>l_{i+1}>l_{i+2}-1>\cdots$.

Now $\delta\left(l_{i+1}-1\right)=\delta(3)=1=\delta(1)=\delta\left(l_{i}\right)$ and $l_{i-1}-l_{i}=l_{i-1}-\left(l_{i+1}+2\right) \geq 3$, so $\lambda$ cannot be constructed from $\tilde{\lambda}$ as in (a).

But also $\delta\left(l_{i+2}-1\right)=\delta(0)=2=\delta(4)=\delta\left(l_{i+1}\right)$ and $l_{i}-l_{i+1}=2$ implies that $\lambda$ cannot be constructed from $\tilde{\lambda}$ as in (b), a contradiction.

(v) Suppose $\lambda$ has a subsequence $\left(l_{i}, l_{i+1}, l_{i+2}\right)=(5 k+6,5 k+4,5 k)$ for some $i$.

Possible predecessors for $\lambda$ in $\mathscr{C}_{5}(n-1) \subseteq \mathscr{D}_{5}(n-1)$ are (a) $\tilde{\lambda}: \cdots l_{i}>$ $l_{i+1}-1>l_{i+2}>\cdots$ and (b) $\tilde{\lambda}: \cdots l_{i}>l_{i+1}>l_{i+2}-1>\cdots$.

In $(\mathrm{a}), \delta\left(l_{i}\right)=\delta(1)=\delta(3)=\delta\left(l_{i+1}-1\right)$ and $l_{i-1}-l_{i}=l_{i-1}-\left(l_{i+2}+2\right) \geq 3$. In (b), $\delta\left(l_{i+1}\right)=\delta(4)=2=\delta\left(l_{i+2}-1\right)$ and $l_{i}-l_{i+1}=2$. So in both cases, $\lambda$ cannot be constructed from $\tilde{\lambda}$.

By similar arguments one can show that $\lambda$ has no subsequence $\left(l_{i}, l_{i+1}\right.$, $\left.l_{i+2}\right)=(5 k+5,5 k+1,5 k-1)$.

(vi) Finally, suppose that $\lambda$ has a subsequence

$$
\left(l_{i}, l_{i+1}, l_{i+2}, l_{i+3}\right)=(5 k+6,5 k+5,5 k, 5 k-1)
$$

for some $i$.

The only possible predecessor in $\mathscr{C}_{5}(n-1) \subseteq \mathscr{D}_{5}(n-1)$ is

$$
\tilde{\lambda}: \cdots l_{i}>l_{i+1}>l_{i+2}>l_{i+3}-1 \text {. }
$$

But $\delta\left(l_{i+3}-1\right)=\delta(3)=1=\delta(1)=\delta\left(l_{i}\right)$ and $l_{i-1}-l_{i}=l_{i-1}-\left(l_{i+1}+1\right) \geq 4$, hence $\lambda$ cannot be constructed from $\tilde{\lambda}$.

Having checked all conditions for $\mathscr{D}_{5}$-partitions, we conclude that none of these can fail for $\lambda$ - contradiction. 
Hence we have proved $\mathscr{C}_{5}(n) \subseteq \mathscr{D}_{5}(n)$.

Before we turn to the proof of the other inclusion we have to recall some definitions (see [BMO, §2]):

If $\lambda=\left(l_{1}, l_{2}, \ldots, l_{m}\right) \vdash n$ has distinct parts, the shifted Young diagram $S(\lambda)$ of $\lambda$ is obtained from the usual Young diagram (Ferrers diagram) by shifting the $i$ th row $(i-1)$ positions to the right. Thus if $\lambda=(7,3,1)$ then

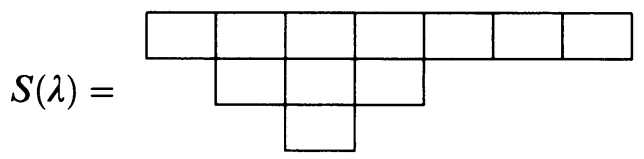

The $j$ th node in the $i$ th row is called the $(i, j)$-node.

The $\overline{5}$-residue of the $(i, j)$-node of $S(\lambda)$ is defined to be

1 if $j \equiv 0$ or $1(\bmod 5)$,

2 if $j \equiv 2$ or $4(\bmod 5)$,

3 if $j \equiv 3(\bmod 5)$.

The $\overline{5}$-residue diagram of $\lambda$ is the diagram obtained from $S(\lambda)$ by replacing the $(i, j)$-node by its $\overline{5}$-residue.

Thus the $\overline{5}$-residue diagram of $\lambda=(7,3,1)$ is

$$
\begin{array}{lllllll}
1 & 2 & 3 & 2 & 1 & 1 & 2 \\
& 1 & 2 & 3 & & & \\
& & 1 & & & &
\end{array}
$$

The $\overline{5}$-residue diagram fits well with the construction rules for $\mathscr{C}_{5}$ as described in $\S 1$. Indeed, if $\lambda=\left(l_{1}, \ldots, l_{m}\right) \in \mathscr{C}_{5}(n-1)$, then the extensions $\tilde{\lambda} \in \mathscr{C}_{5}(n)$ of $\lambda$ correspond to adjoining the highest possible nodes with $\overline{5}$-residue 1,2 , and 3 , respectively. The $\overline{5}$-residues are not equal to the $\delta$ 's occurring in $\S 1$, but it is easy to see that the description above is equivalent to following the construction rules for $\mathscr{C}_{5}(n)$ given in $\S 1$.

For $\lambda=(7,3,1) \in \mathscr{C}_{5}(11)$ its successors in $\mathscr{C}_{5}(12)$ are

$(8,3,1)$ corresponding to adding the highest possible 3-node:

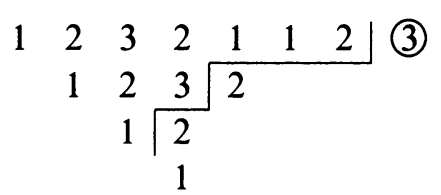

and

$(7,4,1)$ corresponding to adding the highest possible 2-node:

$$
\begin{aligned}
& \begin{array}{lllllll|l}
1 & 2 & 3 & 2 & 1 & 1 & 2 & 3
\end{array} \\
& \begin{array}{llll}
1 & 2 & 3 \\
& 1 & 2
\end{array}
\end{aligned}
$$

but not $(7,3,2)$ since this would correspond to adding a 2-node which is not highest possible. Note also that there is no 1 -node that could be adjoined to $\lambda$. 
We also have to define ladders in the $\overline{5}$-residue diagram:

$\begin{array}{llllllllllllll}1 & 2 & 3 & 2 & 1 & 1 & 2 & 3 & 2 & 1 & 1 & 2 & 3 & 2 \\ & 1 & 2 & 3 & 2 & 1 & 1 & 2 & 3 & 2 & 1 & 1 & 2 & 3 \\ & & 1 & 2 & 3 & 2 & 1 & 1 & 2 & 3 & 2 & 1 & & \\ & & & 1 & 2 & 3 & 2 & 1 & 1 & 2 & & & & \\ & & & & 1 & 2 & 3 & 2 & 1 & & & & & \end{array}$

Here, the ladders are indicated by the lines joining the 1's, 2's, and 3's respectively.

More precisely, for every $r \in\{1,2,3\}$ and $i \geq 1$ we have an $r$-ladder $L_{i, r}$ starting with the first $r$-node in row $i$ and consisting of $r$-nodes only, which are connected as follows:

$$
\begin{aligned}
L_{i, 1}:(i, 1) & \rightarrow(i-2,6) \rightarrow(i-2,5) \rightarrow(i-4,11) \rightarrow(i-4,10) \\
& \rightarrow(i-6,16) \rightarrow(i-6,15) \rightarrow \cdots
\end{aligned}
$$

(ending in row 1 or 2 , depending on $i$ being odd or even)

$$
\begin{aligned}
L_{i, 2}:(i, 2) & \rightarrow(i-1,4) \rightarrow(i-2,7) \rightarrow(i-3,9) \rightarrow \\
& \rightarrow(i-4,12) \rightarrow(i-5,14) \rightarrow \cdots, \\
L_{i, 3}:(i, 3) & \rightarrow(i-2,8) \rightarrow(i-4,13) \rightarrow \cdots
\end{aligned}
$$

(ending in row 1 or 2, depending on $i$ being odd or even).

For a given partition $\lambda$, the ladders $L_{i, r}(\lambda)$ are the parts of these ladders $L_{i, r}$ in (the $\overline{5}$-residue diagram of) $\lambda$.

Proposition 4.3. For all $n \in \mathbb{N}$, we have $\mathscr{D}_{5}(n) \subseteq \mathscr{C}_{5}(n)$.

Proof. We take $\lambda \in \mathscr{D}_{5}(n)$ and show how to construct it by the inductive procedure.

For this, we consider the ladders $L_{i, r}(\lambda)$ in $\lambda$, and work successively from one ladder to the next, from top down (i.e., starting with the highest node in the ladder): $L_{1,1}(\lambda), L_{1,2}(\lambda), L_{1,3}(\lambda), L_{2,1}(\lambda), L_{2,2}(\lambda), \ldots$. This runs smoothly (i.e., in accordance with the $\mathscr{C}_{5}$-construction rules) as long as the ladders $L_{i, r}(\lambda)$ are the top parts of the ladders $L_{i, r}$.

Claim. For $r=1$ or 3 , all the ladders $L_{i, r}(\lambda)$ are at the top of the corresponding ladders $L_{i, r}$, i.e., $L_{i, r}(\lambda)$ consists of the highest $l$ nodes in $L_{i, r}$ for $l=\left|L_{i, r}(\lambda)\right|$.

Proof. First consider the case $r=1$, and assume $L_{i, 1}(\lambda)$ has its lowest 1-node in the position $(j, 5 k)$.

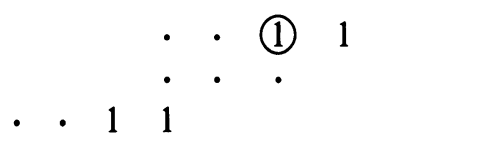

Then $l_{j}=5 k$ and $l_{j-2}-l_{j} \geq 6$ implies $l_{j-2} \geq 5 k+6$ and hence $L_{i, 1}(\lambda)$ contains the 1-nodes in row $j-2$ of $L_{i, 1}$. Now $L_{i, 1}(\lambda)$ must contain all nodes of $L_{i, 1}$ above $(j, 5 k)$. If the lowest 1 -node of $L_{i, 1}(\lambda)$ is in the position $(j, 5 k+1)$, then $l_{j} \geq 5 k+1$ and $l_{j-2}-l_{j} \geq 5$ also give $l_{j-2} \geq 5 k+6$. Hence again $L_{i, 1}(\lambda)$ is a top part of $L_{i, 1}$. 
Now consider a 3-ladder $L_{i, 3}(\lambda)$ with lowest node in the position $(j, 5 k+3)$.

Then $l_{j} \geq 5 k+3$ and $l_{j-2}-l_{j} \geq 5$ imply $l_{j-2} \geq 5 k+8$, and thus the next 3node at position $(j-2,5 k+8)$ is also in $L_{i, 3}(\lambda)$. Again, by the same argument $L_{i, 3}(\lambda)$ contains all 3-nodes of $L_{i, 3}$ above row $j$, hence is a top part of this ladder.

So only the 2-ladders $L_{i, 2}(\lambda)$ are critical, and indeed these may have "holes", but only one:

Claim. For $r=2$, the 2-ladders $L_{i, 2}(\lambda)$ are top parts of $L_{i, 2}$ except that one 2-node (at a position $(j, 5 k+2)$ one row above the lowest node in $L_{i, 2}(\lambda)$ ) may be missing.

Proof. First assume that the lowest node of $L_{i, 2}(\lambda)$ is in a position $(j, 5 k+2)$.

$$
\begin{array}{llll} 
& & 3^{2} & 2 \\
3 & 2 & 2 & \\
& & &
\end{array}
$$

If $l_{j}=5 k+2$, then the exclusion of subsequences $(5 k+3,5 k+2)$ guarantees that the 2-node in position $(j-1,5 k+4)$ belongs to $L_{i, 2}(\lambda)$. If $l_{j}>5 k+2$, then $l_{j-1}>5 k+3$ and this also forces this 2-node to belong to $L_{i, 2}(\lambda)$.

Furthermore, $l_{j-2}-l_{j} \geq 5$ leads to $l_{j-2} \geq 5 k+7$, hence also the 2-node in position $(j-2,5 k+7)$ is in $L_{i, 2}(\lambda)$. Continuing like this, we see that $L_{i, 2}(\lambda)$ is indeed a top part of $L_{i, 2}$.

Thus we may now assume that the lowest 2-node in $L_{i, 2}(\lambda)$ is in position $(j, 5 k+4)$.

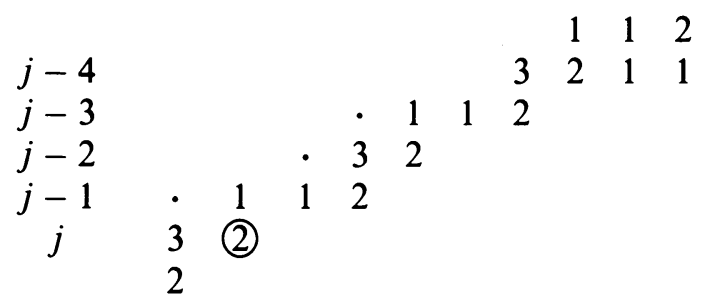

Again, $l_{j-2}-l_{j} \geq 5$ gives the 2 -node in position $(j-2,5 k+9)$, and similarly we get all 2-nodes in position $(j-2 m, 5(k+m)+4)$. As $l_{j-1} \geq 5 k+5, L_{i, 1}(\lambda)$ contains the 1-node at position $(j-1,5 k+5)$ and hence, by the previous arguments, all 1 -nodes in $L_{i, 1}$ above this node.

Now suppose that the 2-node in position $(j-3,5(k+2)+2)$ is missing in $L_{i, 2}(\lambda)$. As subsequences of the form $(5(k+2)+4,5(k+2)+1,5(k+1)+4)$ and $(5(k+3), 5(k+2)+1,5(k+1)+4)$ cannot occur in $\lambda \in \mathscr{D}_{5}(n)$, and by assumption $l_{j-3}=5(k+2)+1$, we conclude (using the previous arguments 
again): $l_{j-4} \geq 5(k+3)+1$, i.e., the next 1-ladder $L_{(i+1), 1}(\lambda)$ must contain all 1 -nodes in $L_{(i+1), 1}$ down to row $j-4$. Furthermore, as $l_{j-5} \geq 5(k+3)+2$, this forces $L_{i, 2}(\lambda)$ to contain all 2-nodes down to row $j-3$. Hence we know now that at most the 2-nodes in positions $(j-3,5(k+2)+2)$ and $(j-1,5(k+1)+2)$ can constitute holes in $L_{i, 2}(\lambda)$. far:

Still assuming we have these two 2-nodes missing in $L_{i, 2}(\lambda)$, we know so

$$
\begin{aligned}
& l_{j-3}=5(k+2)+1 ; \quad l_{j-2}=5(k+1)+4 \text { or } 5(k+2) ; \\
& l_{j-1}=5(k+1) \text { or } 5(k+1)+1 ; 5 k+4 \leq l_{j}<l_{j-1} .
\end{aligned}
$$

As $\lambda \in \mathscr{D}_{5}(n)$, the following subsequences are all forbidden:

$$
\begin{array}{lcll}
5(k+2)+1, & 5(k+2), & 5(k+1), & 5 k+4 \\
5(k+1)+4, & 5(k+1)+1, & 5 k+4 \\
5(k+2), & 5(k+1)+1, & 5 k+4 \\
5(k+2), & *, & 5(k+1) \\
5(k+1)+4, & *, & 5(k+1) \\
5(k+2)+1, & 5(k+1)+4, & 5(k+1)
\end{array}
$$

But then there is no possibility for $l_{j-3}, l_{j-2}, l_{j-1}, l_{j}$ left, contradiction!

Hence it follows that $L_{i, 2}(\lambda)$ can have at most one hole at position $(j-$ $1,5 k+7)$.

Now if $L_{i, 2}(\lambda)$ has no hole or if it has a hole at position $(j-1,5 k+7)$ but this is not "accessible", i.e., $l_{j-1}=5 k+5$, then we can build up $\lambda$ along $L_{i, 2}(\lambda)$ from top down without any problem.

So assume now $l_{j-1}=5 k+6$.

We give a procedure that constructs all 2-nodes in $L_{i, 2}(\lambda)$ according to the $\mathscr{C}_{5}$-rules. We may assume that the part of $\lambda$ up to and including $L_{i-1,3}(\lambda)$ has already been constructed.

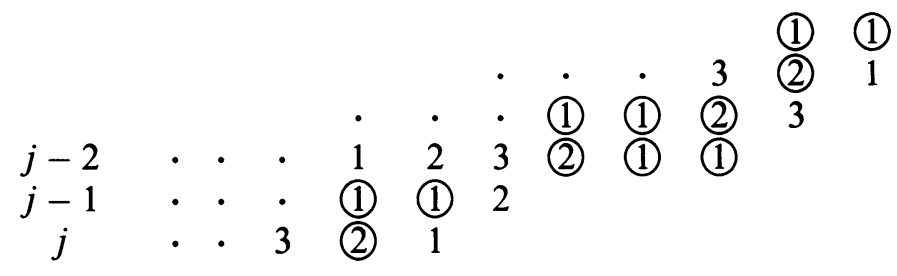

As $(5 k+9,5 k+6,5 k+4)$ and $(5 k+10,5 k+6,5 k+4)$ are forbidden, we must have $l_{j-2} \geq 5 k+11$, and hence $L_{i+1,1}(\lambda)$ contains all 1-nodes in $L_{i+1,1}$ down to row $j-2$.

Now we continue with the construction of $\lambda$ : first add all the nodes in $L_{i, 1}(\lambda)$ from top down up to the 1 -node in position $(j-1,5 k+5)$. Now we may add all 2-nodes in $L_{i, 2}(\lambda)$, as the 2-node in position $(j-1,5 k+7)$ is not accessible and hence the 2-node in position $(j, 5 k+4)$ can be added according to the $\mathscr{C}_{5}$-rules. Next add the nodes in $L_{i, 3}(\lambda)$, and then the nodes in $L_{i+1,1}(\lambda)$ down to row $j-2$. At this step, the 1 -node in $L_{i, 1}(\lambda)$ in position $(j-1,5 k+6)$ is the highest accessible 1 -node, hence it can be added according to the $\mathscr{C}_{5}$-rules. If $L_{i+1,1}(\lambda)$ has a further node in row $j$, we now add this. Then we continue to add the 1-nodes on $L_{i, 1}(\lambda)$ to its bottom. If $L_{i+1,1}(\lambda)$ has no node in row 
$j$, then there are no further nodes in $L_{i, 1}$. Having completed all these steps, we have now constructed the part of $\lambda$ up to and including $L_{i+1,1}(\lambda)$. Then we continue by working down the ladders as before, and as described above, in the case of a hole in a 2-ladder at a position $(j, 5 k+2)$, the "short route" between the two 1-nodes in row $j$ preceding this 2-node is replaced by a deviation through the next three ladders, but in higher rows. Note that it might already be necessary to insert such a deviation in the construction of $L_{i+1,1}(\lambda)$, but this can only occur in rows $\leq j-3$.

This finishes the algorithm for constructing $\lambda$ as a $\mathscr{C}_{5}$-partition. Hence we have now proved $\mathscr{D}_{5}(n) \subseteq \mathscr{C}_{5}(n)$, and thus we have also completed the proof of the theorem.

\section{ACKNOWLEDGMENT}

The first author was supported by National Science Foundation Grant DMS 8702695-04. The second author is grateful to the Deutsche Forschungsgemeinschaft for their support through the Heisenberg program, grant no. Be 923/4-1, and to the Institute for Experimental Mathematics at the University of Essen for its hospitality.

\section{REFERENCES}

[A1] G. E. Andrews, A generalization of the classical partition theorems, Trans. Amer. Math. Soc. 145 (1969), 205-221.

[A2] _ On a theorem of Schur and Gleissberg, Arch. Math. 22 (1971), 165-167.

[A3] - On the general Rogers-Ramanujan theorem, Mem. Amer. Math. Soc. 152 (1974).

[A4] _ q-series, CBMS Regional Conf. Ser. in Math., vol. 66, Amer. Math. Soc., Providence, RI, 1986.

[A5] _ Physics, Ramanujan and computer algebra, Computer Algebra (D. V. Chudnovsky and R. D. Jenks, eds.), Dekker, New York, 1989, pp. 97-109.

[BMO] C. Bessenrodt, A. O. Morris, and J. B. Olsson, Decomposition matrices for spin characters of symmetric groups at characteristic 3, preprint, 1992.

[F] W. Feit, The representation theory of finite groups, North-Holland, Amsterdam, 1982.

[JK] G. James and A. Kerber, The representation theory of the symmetric group, Addison-Wesley, London, 1981.

[MY] A. O. Morris and A. K. Yaseen, Decomposition matrices for spin characters of symmetric groups, Proc. Roy. Soc. Edinburgh Sect. A 108 (1988), 145-164.

[NT] H. Nagao and Y. Tsushima, Representations of finite groups, Academic Press, San Diego, 1989.

[S1] I. Schur, Über die Darstellung der symmetrischen und der alternierenden Gruppe durch gebrochene lineare Substitutionen, J. Reine Angew Math. 139 (1911), 155-250 (Ges. Abhandlungen 1, Springer-Verlag, 1973, pp. 346-441).

[S2] I. Schur, Zur additiven Zahlentheorie, Sitzungsber. Preuss. Akad. Wiss., Phys.-Math. K1. (1926), 488-496 (Ges. Abhandlungen 3, Springer-Verlag, 1973, pp. 43-50).

Department of Mathematics, Pennsylvania State University, University Park, PennSYLVANIA 16802

E-mail address: andrews@math.psu.edu

FAKUltät FÜr MAthematik, Technische Universität, 39016 Magdeburg, GermaNY

E-mail address: bessen@dmdtu11.urz.tu-magdeburg.de

MAtematisk Institut, Universitetsparken 5, 2100 COPEnhagen 0, DenmaRk

E-mail address: olsson@math.ku.dk 